\title{
La relation entre Autochtones et gouvernements provinciaux vue à la lumière du développement nordique au Québec et en Ontario
}

\section{What Northern Development Strategies Can Tell About the Provinces' Relationship with First Peoples: A Case Study of Ontario and Québec \\ La relación entre indígenas y gobiernos provinciales a la luz del desarrollo del Norte en Quebec y Ontario}

\section{Alexandre Germain}

Volume 41, numéro 1, 2011

Plan nord, éducation et droit

URI : https://id.erudit.org/iderudit/1012711ar

DOI : https://doi.org/10.7202/1012711ar

Aller au sommaire du numéro

Éditeur(s)

Recherches amérindiennes au Québec

ISSN

0318-4137 (imprimé)

1923-5151 (numérique)

Découvrir la revue

Citer cet article

Germain, A. (2011). La relation entre Autochtones et gouvernements provinciaux vue à la lumière du développement nordique au Québec et en Ontario. Recherches amérindiennes au Québec, 41(1), 91-95.

https://doi.org/10.7202/1012711ar
Résumé de l'article

Le Québec n'est pas la seule province canadienne à se tourner vers le Nord pour assurer son développement économique. L’Ontario a dévoilé des pans importants de sa stratégie nordique peu avant la publication du Plan Nord du gouvernement du Québec. Cet article propose une analyse synthétisée des principaux documents définissant l'approche des deux gouvernements et fait une comparaison de leur vision respective du développement, de la relation au territoire qu'ils suggèrent et de la relation entre les Autochtones et l'État qui est mise de l'avant par chacun. Il en ressort des différences d'ampleur, de moyens, de démarche, d'acteurs et de relations qui semblent avoir un lien avec la relation qu'entretient chacune des deux provinces avec le fédéralisme canadien. En d'autres mots, l'influence du fédéralisme canadien sur les stratégies de développement nordique provinciales et sur les relations avec les Autochtones n'est pas uniforme. 


\section{Alexandre}

Germain

Université du

Québec à Montréal

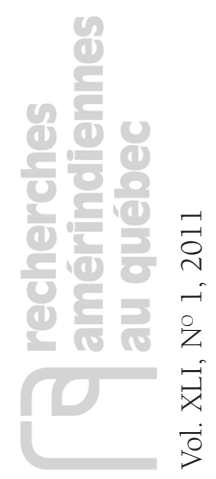

E QUÉBEC ET L'ONTARIO disposent
chacun d'un important terri-
toire nordique peu développé en raison principalement de la difficulté d'y accéder. La conjoncture économique mondiale, qui laisse entrevoir une importante croissance de la demande en ressources naturelles de la part des économies émergentes, crée les conditions propices à une poussée du développement vers le Nord. C'est dans ce contexte que les gouvernements du Québec et de l'Ontario ont dévoilé, quasi simultanément, leurs nouvelles politiques de développement nordique.

Au Canada, le Nord est cette région où, presque par définition, il se produit une rencontre entre Autochtones et non-Autochtones. Les politiques de développement nordique sont donc des enjeux clés dans l'étude des relations entre les Autochtones et les États. La simultanéité des démarches québécoise et ontarienne incite à en faire la comparaison. Lintérêt de cette comparaison réside dans la profonde différence du rapport de chacune de ces provinces avec la fédération canadienne, alors que leurs gouvernements émanent de deux majorités nationales différentes (en présumant que l'identité nationale majoritaire en Ontario est canadienne).

Ce texte actualise, en les schématisant, les grandes lignes d'une comparaison des approches québécoise et ontarienne, décrite plus longuement dans une publication spéciale du réseau DIALOG (Germain 2012 ${ }^{1}$ ). Notre objectif est ici de faire ressortir, par contraste, les spécificités de l'approche québécoise telle que suggérée par le Plan Nord. Pour faciliter sa compréhension, l'information a été synthétisée à l'aide de deux tableaux et est accompagnée d'un court texte d'interprétation des principales différences entre les deux approches.

Nous invitons donc le lecteur à porter une attention spéciale à ces tableaux: le premier synthétise les plans de développement nordique de l'Ontario et du Québec et le deuxième identifie des éléments contrastants. Mentionnons que, alors que la politique nordique du Québec est redéfinie dans un document intitulé Plan Nord. Faire le Nord ensemble: le chantier d'une génération (Québec 2011), la nouvelle politique ontarienne s'appuie sur deux documents complémentaires: Loi relative à l'aménagement et à la protection du Grand Nord - « Ontario Far North 
Tableau 1

\section{Synthèse des plans de développement nordique de l'Ontario et du Québec}

\begin{tabular}{|c|c|c|c|}
\hline $\begin{array}{l}\text { PLAN DE DÉVELLOPPEMENT } \\
\text { NORDIQUE }\end{array}$ & $\begin{array}{l}\text { LOI RELATIVE À L'AMÉNAGEMENT ET À LA } \\
\text { PROTECTION DU GRAND NORD DE L'ONTARIO } \\
\text { (ONTARIO FAR NORTH ACT) }\end{array}$ & $\begin{array}{l}\text { PLAN DE CROISSANCE DU NORD } \\
\text { DE L'ONTARIO }\end{array}$ & $\begin{array}{l}\text { Plan Nord. Faire le NoRd ensemble : le CHAN- } \\
\text { tIER D'UNE GÉNÉRATION (QuUÉBEC) }\end{array}$ \\
\hline Date de publication & 25 octobre 2010 & 3 mars 2011 & 9 mai 2011 \\
\hline $\begin{array}{l}\text { Population du } \\
\text { territoire visé }\end{array}$ & $\begin{array}{l}24000 \text { personnes, dont environ } \\
22000 \text { autochtones des nations crie } \\
\text { et ojibwa }\end{array}$ & $\begin{array}{l}800000 \text { personnes, dont environ } \\
140000 \text { francophones et } \\
100000 \text { autochtones }\end{array}$ & $\begin{array}{l}120000 \text { personnes, dont au moins } \\
33000 \text { autochtones des nations crie, inuite, } \\
\text { innue et naskapie (les Algonquins et les } \\
\text { Attikameks ne sont pas mentionnés) }\end{array}$ \\
\hline Nature du document & Loi provinciale & $\begin{array}{l}\text { Énoncé de politiques publiques et plan } \\
\text { quinquennal }\end{array}$ & $\begin{array}{l}\text { Énoncé de vision, document promotionnel et } \\
\text { plan quinquennal }\end{array}$ \\
\hline $\begin{array}{l}\text { Démarche } \\
\text { d'élaboration }\end{array}$ & $\begin{array}{l}\text { Consultative et délibérative (en assemblée } \\
\text { législative), sous l'égide du ministère des } \\
\text { Richesses naturelles }\end{array}$ & $\begin{array}{l}\text { Consultative, sous l'égide du ministère de } \\
\text { l'Infrastructure et du ministère du } \\
\text { Développement du Nord, des Mines et } \\
\text { des Forêts }\end{array}$ & $\begin{array}{l}\text { Consultative et partenariale (en tables de négo- } \\
\text { ciation), sous l'égide d'un comité ministériel } \\
\text { présidé par le ministère des Ressources natu- } \\
\text { relles et de la Faune }\end{array}$ \\
\hline & $\begin{array}{l}\text { et les écosystèmes dans le Grand Nord en } \\
\text { incluant une superficie d'au moins } \\
225000 \text { kilomètres carrés dans un réseau } \\
\text { interrelié de zones protégées désignées } \\
\text { dans les plans communautaires } \\
\text { d'aménagement du territoire » } \\
\text { 3) « Maintenir la diversité biologique, les } \\
\text { processus et fonctions écologiques, y } \\
\text { compris le stockage et la séquestration du } \\
\text { dioxyde de carbone dans le Grand Nord » } \\
\text { 4) " Permettre un développement } \\
\text { économique durable qui profite aux } \\
\text { Premières nations » }\end{array}$ & $\begin{array}{l}\text { 2) Favoriser un processus rationnel et } \\
\text { équilibré de prise de décision } \\
\text { 3) Permettre une croissance planifiée de } \\
\text { manière large et intégrée } \\
\text { 4) Définir une vision à long terme et } \\
\text { prévoir la coordination des politiques de } \\
\text { croissance entre tous les niveaux de } \\
\text { gouvernement }\end{array}$ & $\begin{array}{l}\text { développement durable qui intègre le déve- } \\
\text { loppement énergétique, minier, forestier, } \\
\text { bioalimentaire, touristique et du transport, la } \\
\text { mise en valeur de la faune ainsi que la } \\
\text { protection de l'environnement et la } \\
\text { conservation de la biodiversité. II favorisera le } \\
\text { développement au bénéfice des commu- } \\
\text { nautés concernées et du Québec tout entier, } \\
\text { et ce, dans le respect des cultures et des } \\
\text { identités. » (p. 14) }\end{array}$ \\
\hline
\end{tabular}

Act »(Ontario 2010) et Plan de croissance du Nord de l'Ontario (Ontario 2011). Notre analyse porte sur ces trois textes. Le premier tableau présente de manière synthétique la portée et le contenu de ces textes, sans entrer dans les détails (voir le texte d'Hugo Asselin, dans ce numéro, pour une discussion critique de la place des Autochtones dans le Plan Nord québécois). Le deuxième tableau propose un aperçu des principales différences d'approche entre le Québec et l'Ontario.

Aux fins de la comparaison, nous avons identifié trois ordres de questionnement portant sur les politiques de développement nordique du Québec et de l'Ontario.
Concernant d'abord la vision du développement nordique, les approches québécoise et ontarienne présentent des différences d'ampleur (projet de société vs planification territoriale), de moyens (création de Société d'État et investissement majeurs vs principes directeurs et adoption d'une loi), de démarche (structure complexe avec beaucoup d'intervenants vs petites équipes sous l'autorité du ministre) et d'inclusion du gouvernement fédéral (absent vs présent). Il semble que ces différences peuvent être interprétées en parallèle avec les relations qu'entretiennent les sociétés québécoise et ontarienne avec « leur» territoire nordique. 
Faut-il rappeler que le Nord occupe une place considérable tant dans les imaginaires nationaux québécois que canadien. Au Québec, où la société majoritaire est d'origine canadienne-française, le Nord fut un thème récurrent de la littérature (Warwick 1972) et de la pensée chez une certaine élite intellectuelle (Morissonneau 1978). Il figure comme l'un des mythes de la reconquête du Nouveau Monde, par lequel la nation assumerait son destin historique (Bouchard 2004 : 56). Plusieurs éléments de discours du Plan Nord laissent transparaître que l'on fait appel à cet imaginaire national. Citons par exemple le premier paragraphe de la conclusion du document Plan Nord tel qu'il a été rendu public le 9 mai 2011 :

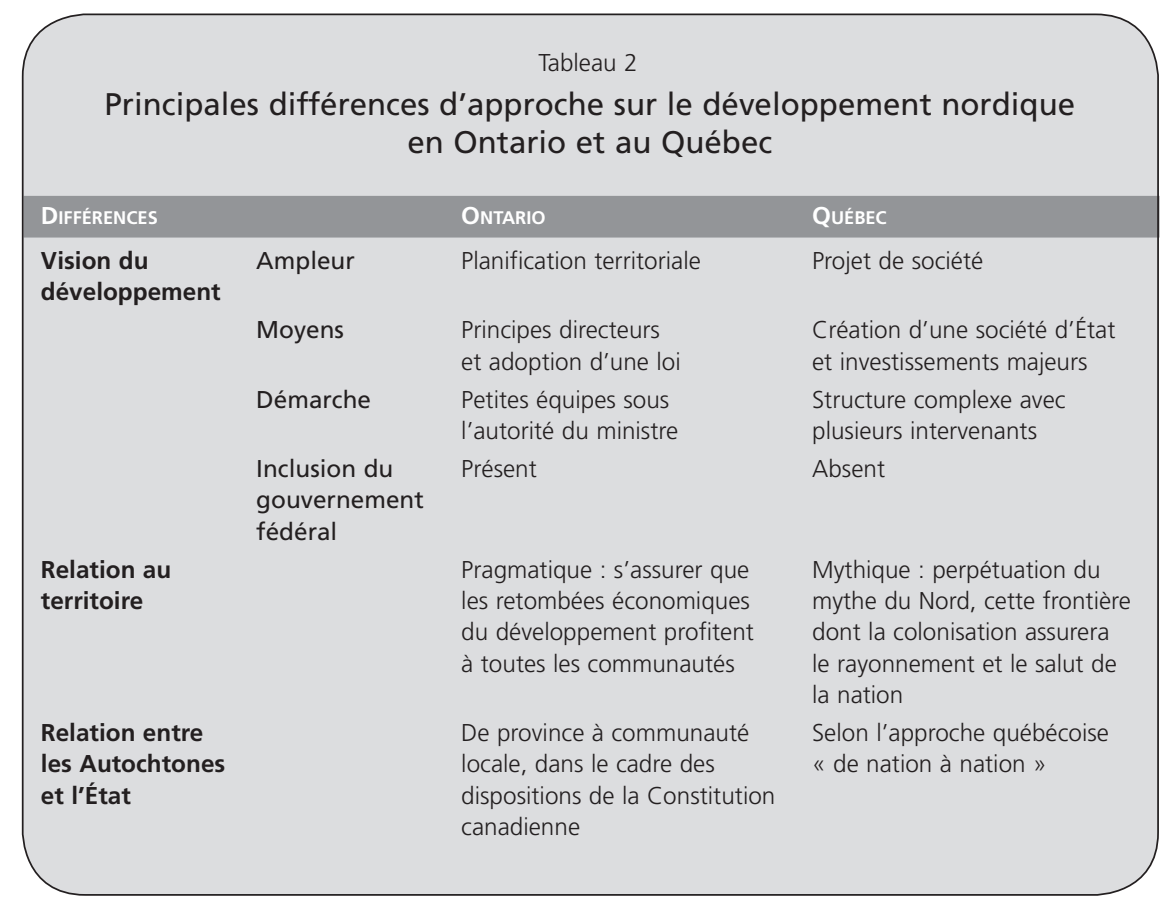

Le Nord fait partie de l'identité québécoise. Depuis toujours, il nourrit notre imaginaire. II a marqué notre littérature et notre culture; il a façonné notre économie, nous y puisons la majeure partie de notre énergie... Et aujourd'hui, avec le Plan Nord, nous nous préparons à faire du Nord un exemple d'envergure internationale de développement durable à la faveur d'un partenariat respectueux et moderne avec les Premières Nations, les Inuits et les communautés locales. (Québec 2011 : 119)

En comparaison, ce Nord qui est érigé en «mythe national » canadien (Berger 1966:22) et vu comme le «fondement de la communauté imaginée » (Grace 2002 : 23) ne suscite aucun écho dans la politique ontarienne. Il est fort possible que cette différence s'explique par le fait que, le territoire national canadienfrançais étant désormais réduit aux frontières du Québec, la projection nordique de l'imaginaire national québécois s'exerce dans les limites de la province. Lidentité nationale en Ontario revendique des frontières plus vastes que celles de la province, et il apparaît ainsi plus normal que le mythe nordique canadien ne se manifeste pas, ou très peu, dans la politique ontarienne de développement nordique. Autrement dit, on ne retrouve pas, dans la politique ontarienne, la portée identitaire donnée au Plan Nord québécois, ce «chantier d'une génération» qui semble bien perpétuer un mythe du Nord où la colonisation de «notre dernière grande frontière du Nord » (Robitaille 2008) est essentielle au salut de la nation (par son succès économique, dans le cas présent). Ainsi, à l'instar de Caroline Desbiens (voir notamment son texte cosigné avec Étienne Rivard et publié dans ce numéro), on peut croire que la relation au territoire des sociétés majoritaires québécoise et ontarienne a une incidence sur la relation entre leurs gouvernements et les Autochtones.

Dans les Nords mythifiés par les acteurs sociaux et politiques du Sud, l'autochtone est plutôt absent ou instrumentalisé. Les peuples autochtones ne peuvent néanmoins plus être exclus du développement nordique et de fait, une place importante leur est accordée dans les politiques de développement nordique du Québec et de l'Ontario. Un chapitre leur est d'ailleurs consacré dans le Plan de croissance, et leur présence sur le territoire est en quelque sorte la raison d'être de la loi ontarienne relative à l'aménagement et à la protection du territoire. Ils sont en outre constamment cités dans le Plan Nord, en plus des pages consacrées à la «relation avec les Premières Nations et les Inuits basée sur le respect mutuel et le partenariat »(Québec 2011 : 23-25) et du souci réitéré dans les priorités d'actions du gouvernement pour leurs besoins socio-économiques (voir l'annexe E du Plan Nord).

Les gouvernements du Québec et de l'Ontario se proposent de développer une approche partenariale avec les Autochtones. Cependant, l'approche qui se dégage de la loi ontarienne sur l'aménagement et la protection du Grand Nord met de l'avant l'idée d'établir des partenariats qui font seulement appel à la participation des communautés locales. Chaque communauté ou regroupement de communautés peut s'engager dans l'aménagement de son territoire par l'entremise d'une équipe mixte de planification ou d'un organisme paritaire, où elle se trouvera à travailler avec des fonctionnaires. Dans ce modèle, le pouvoir d'avaliser toute décision revient aux conseils de 
bande. Cette approche s'apparente au modèle de la cogestion des ressources naturelles, de plus en plus répandu au Canada. Lapproche québécoise propose plutôt d'établir des partenariats s'inscrivant dans la logique d'une relation de nation à nation, où la cogestion n'est pas nécessairement mise à l'avant-plan. Par contre, le pouvoir décisionnel autochtone ne se retrouve pas qu'au niveau local.

Ces particularités sont à mettre en perspective avec la relation qu'entretient chacune des provinces avec la fédération canadienne. Dans un texte portant sur l'idéal fédéral et l'évolution du système politique canadien, François Rocher a montré que « la compréhension dominante dans la littérature d'expression anglaise a évacué toute référence à la notion d'autonomie au profit de celle d'efficacité, alors que celle que nous retrouvons au sein des travaux produits par les chercheurs québécois francophones et les pratiques gouvernementales qui s'en inspirent ont [sic] fait une place congrue à la notion d'interdépendance », au profit de celle d'autonomie (2006 : 113). Il semble que les positions du Québec et de l'Ontario soient archétypiques de leur relation avec le fédéralisme canadien. Autrement dit, la grande préoccupation pour l'autonomie, au Québec, dicte au gouvernement une approche de nation à nation qui néglige l'interdépendance avec les nations autochtones alors que celle pour l'efficacité, en Ontario, suggère au gouvernement une approche de cogestion qui néglige l'autonomie des Premières Nations. D'ailleurs, le Plan de croissance a été critiqué pour ses lacunes eu égard à la consultation, et la loi ontarienne n'a pas reçu l'aval du Conseil de la Nation Nishnawbe Aski, dont l'opposition relève du fait qu'elle estime que les communautés qu'elle représente n'exercent pas suffisamment de contrôle sur leur territoire et que l'obligation de protéger $50 \%$ du territoire peut faire obstacle à leur droit au développement ${ }^{2}$.

Quant au Plan Nord, ses auteurs affirment respecter les ententes conclues et vantent l'approche partenariale par laquelle le gouvernement et les représentants autochtones se sont assis aux «tables des partenaires autochtones». Ainsi, on peut supposer que l'approche québécoise semble satisfaire le besoin d'autonomie des Autochtones qui ont signé les conventions nordiques puisque Cris, Inuits et Naskapis ont adhéré au Plan Nord. Par ailleurs, sur les huit communautés innues dont les villages se trouvent au nord du $49^{e}$ parallèle, seules les deux qui ont signé avec les gouvernements une entente de principe sur leurs revendications territoriales ont signifié leur adhésion à ce plan. Les communautés dissidentes (au nombre de cinq), rassemblées au sein de l'Alliance stratégique innue, représentent un cas particulier puisque leur opposition vient davantage d'un problème de reconnaissance de droits lié à l'absence d'entente portant sur leurs revendications territoriales globales que d'un conflit au sujet de la vision du développement. Les difficultés qu'éprouve le gouvernement à régler ce conflit sont d'ailleurs liées au fait que ce qui apparaît comme une absence d'unité chez les Innus pose problème pour la relation de nation à nation qu'il préconise.

Une lecture plus attentive du Plan Nord révèle toutefois, malgré cette sensibilité à l'autonomie «nationale » autochtone, des formulations qui trahissent souvent l'identité d'un concepteur unique, le gouvernement du Québec. Par exemple, on y définit la « relation avec les Premières Nations et les Inuits» (Gouvernement du Québec 2011 : 23, nous soulignons) et non la relation entre le gouvernement et les Premières Nations et les Inuits. Bref, même s'il est question d'un territoire dont la plus grande superficie est habitée en majorité par des autochtones, le Plan Nord, c'est avant tout le plan du gouvernement du Québec, et non celui des Cris, des Innus, des Naskapis ou des Inuits. La société Makivik a d'ailleurs cru bon de répliquer avec son Plan Nunavik ${ }^{3}$ (voir le texte de Jean-François Arteau dans ce numéro). Ici, il semble que la relation qu'entretient le Québec avec « son » Nord, ainsi que l'importance qu'il accorde à sa propre autonomie politique et économique, l'ait rendu moins sensible à la question de l'interdépendance qui le lie aux Autochtones. C'est d'ailleurs dans cette interdépendance que se trouve tout le sens de la citation de Louis-Edmond Hamelin, «Il n'y a pas de vrai Québec sans la zone nordique », placée en exergue du document et citée hors de son contexte, ce qui en brouille le sens. Dans cette perspective, le gouvernement du Québec aurait dû entamer un processus de " co-élaboration », ou « co-création », par lequel le Plan Nord aurait aussi été le fruit d'une initiative des Autochtones. Une telle approche aurait été davantage réalisable si les Autochtones bénéficiaient d'une certaine représentation dans les institutions démocratiques québécoises.

Ainsi, et nous conclurons là-dessus, il est possible de tracer certains parallèles entre la relation qu'entretiennent les gouvernements québécois et ontarien avec les peuples autochtones et avec la fédération canadienne. D’abord par rapport aux institutions fédérales : le Québec, lui-même assez jaloux de son autonomie, exprime une certaine sensibilité pour l'autonomie autochtone dans son approche basée sur un partenariat de nation à nation, mais il ne leur offre pas vraiment la possibilité d'être les « co-concepteurs » du projet de société proposé par son gouvernement. Cette situation suggère que la notion d'interdépendance des nations autochtones et québécoise pourrait être cultivée davantage. En matière de relations avec les Autochtones, l'Ontario développe pour sa part une relation plus alignée sur l'approche canadienne (laquelle est plus sensible à la question de l'interdépendance, 
notamment pour des raisons d'unité nationale) qui fait appel à la cogestion des ressources naturelles, avec les conseils de bande intéressés, et s'applique à respecter ses obligations, définies par la Constitution du pays, à l'égard des Autochtones. Cette approche n'a toutefois pas encore obtenu l'aval du Conseil de la Nation Nishnawbe Aski, qui représente, pour l'essentiel, toutes les communautés nordiques ontariennes. En ce sens, on peut croire que le gouvernement ontarien était davantage préoccupé par des questions d'efficacité que par l'autonomie des communautés autochtones. Ensuite, en rapport avec la relation au territoire, la fédération canadienne permet à une diversité d'imaginaires nordiques (et nationalistes) distincts, et parfois concurrents, de s'exprimer et d'influencer les stratégies nordiques des différents gouvernements - et leurs relations avec les Autochtones, qui en découlent. Linfluence du fédéralisme canadien sur le développement nordique des provinces et sur les relations avec les Autochtones n'est donc pas uniforme et contribue à produire une diversité d'approches au niveau provincial. Cette diversité peut être bonne en soi, mais on n'y trouve pas encore une solution qui puisse vraiment permettre de mettre de l'avant l'autonomie des nations minoritaires les nations autochtones au premier chef.

\section{Notes}

1. La description des approches québécoise et ontarienne y est plus détaillée et la discussion est inscrite dans le débat portant sur les possibilités d'innovation offertes par le fédéralisme canadien, en lien avec son caractère multinational.

2. Se référer aux communiqués de presse de la Nishnawbe Aski Nation parus les 2 et 3 juin 2009, le 13 août 2010 et les 17 et 22 septembre 2010, en ligne au <http://www.nan.on.ca/article/ the-latest-news-from-nan-8.asp >.

3. Une version trilingue du Plan Nunavik est disponible sur le site de l'Administration régionale Kativik à l'adresse suivante: <http://www.krg.ca/images/stories/docs/Communiques_en/ Plan\%20Nunavik.pdf>.

\section{Ouvrages cités}

BERGER, Carl, 1966: "The True North Strong and Free », in Peter Russell (dir.), Nationalism in Canada: 3-26. McGrawHill, Toronto.

BOUCHARD, Gérard, 2004 : La pensée impuissante. Échecs et mythes nationaux canadiens-français (1850-1960). Boréal, Montréal.

GERMAIN, Alexandre, 2012: « Le développement nordique au Québec et en Ontario. Différentes perspectives d'avenir pour les peuples autochtones ». Publication spéciale du Réseau DIALOG (à paraître).

GRACE, Sherrill E., 2001 : Canada and the Idea of North. McGillQueen's University Press, Montréal et Kingston.

MORISSONNEAU, Christian, 1978 : La Terre promise : le mythe du Nord québécois. Hurtubise HMH, Montréal.

ONTARIO (Gouvernement de 1'), 2010: Loi relative à l'aménagement et à la protection du Grand Nord. Disponible sur Internet : <http://www.e-laws.gov.on.ca/html/source/statutes/french/2010/ elaws_src_s10018_f.htm> (consulté le 12 juin 2012).

_, 2011 : Plan de croissance du Nord de l'Ontario. Place à la croissance: de meilleurs choix pour l'avenir. Ministère de l'Infrastructure et ministère du Développement du Nord, des Mines et des Forêts, Ottawa. Disponible sur Internet: <https://www.placestogrow.ca/images/pdfs/GPNO-final-FR.pdf> (consulté le 12 juin 2012).

QUÉBEC (Gouvernement du), 2011 : Plan Nord. Faire le Nord ensemble: le chantier d'une génération. Premier plan d'action 2011-2016. Ministère des Ressources naturelles et de la Faune, Québec. Disponible sur Internet : <http://www.plannord.gouv. qc.ca/documents/plan-action.pdf> (consulté le 12 juin 2012).

ROBITAILLE, Antoine, 2008 : « Charest mise sur le Nord ». Le Devoir, 29 septembre. Disponible sur Internet : <http://www.ledevoir. $\mathrm{com} /$ politique/quebec/208131/charest-mise-sur-le-nord> (consulté le 12 juin 2012).

ROCHER, François, 2006 : « La dynamique Québec-Canada ou le refus de l'idéal fédéral », in Alain-G. Gagnon (dir.), Le fédéralisme canadien contemporain. Fondements, traditions, institutions : 93-146. Presses de l'Université de Montréal, Montréal.

WARWICK, Jack, 1972 : L'appel du Nord dans la littérature canadienne-française. Hurtubise HMH, LaSalle. 